\title{
ESTUDIO COMPARATIVO DE MARCADORES BIOQUÍMICOS DE SALUD, EN DOS GRUPOS ETARIOS DE DOCENTES UNIVERSITARIOS, 2017
}

\author{
COMPARATIVE STUDY OF BIOMARKERS IN TWO AGE GROUPS OF UNIVERSITY PROFESSORS, 2017 \\ Nicanor Domínguez-Navarrete ${ }^{1, a, b}$, Cecilia Rojas-Guerrero ${ }^{1, c}$, Carola Chambers-Medina ${ }^{1, d}$
}

\begin{abstract}
RESUMEN
Objetivo: Comparar los valores de los marcadores bioquímicos sanguíneos: ácido úrico, creatinina, glucosa, colesterol y triglicéridos, entre dos grupos de docentes universitarios aparentemente sanos. Métodos: El primer grupo, formado por 58 docentes de 60 a 64 años y, el segundo por 35 docentes de 75 a 81 años; para evaluar si hay diferencia estadística significativa entre ambos grupos. Resultados: Los valores de ácido úrico en el grupo I estuvieron entre $2,50 \mathrm{mg} / \mathrm{dL}$. y $8,20 \mathrm{mg} / \mathrm{dL}$, y en el grupo II entre $2,50 \mathrm{mg} / \mathrm{dL}$ y $8,63 \mathrm{mg} / \mathrm{dL}$, con diferencia significativa ( $p=0.035$ ). Los valores de creatinina en el grupo I estuvieron entre $0,64 \mathrm{mg} / \mathrm{dL}$ y $1,56 \mathrm{mg} / \mathrm{dL}$, y en el grupo II entre $0,80 \mathrm{mg} / \mathrm{dL}$ y $1,61 \mathrm{mg} / \mathrm{dL}$., con diferencia significativa $(p=0.004)$. Los valores de glucosa en el grupo I estuvieron entre $63 \mathrm{mg} / \mathrm{dL}$ y $188 \mathrm{mg} / \mathrm{dL}$, y en el grupo II entre $60 \mathrm{mg} / \mathrm{dL}$ y $297 \mathrm{mg} / \mathrm{dL}$, sin encontrar diferencia significativa ( $p=0.671$ ). Los valores de colesterol en el grupo I estuvieron entre $125 \mathrm{mg} / \mathrm{dL}$ y $265 \mathrm{mg} /$ $\mathrm{dL}$, y en el grupo II entre $107 \mathrm{mg} / \mathrm{dL}$ y $244 \mathrm{mg} / \mathrm{dL}$, sin encontrar diferencia significativa $(p=0.053$ ). Los valores de triglicéridos en el grupo l estuvieron entre $58 \mathrm{mg} / \mathrm{dL}$ y $507 \mathrm{mg} / \mathrm{dL}$, y en el grupo II entre $68 \mathrm{mg} / \mathrm{dL}$ y $314 \mathrm{mg} /$ $\mathrm{dL}$, sin encontrar diferencia significativa $(\mathrm{p}=0.104)$. Conclusión: En los marcadores bioquímicos de ácido úrico y creatinina hay diferencia significativa entre ambos grupos etarios. En cambio, en los marcadores de glucosa, colesterol y triglicéridos, no se demuestra diferencia significativa entre ambos grupos etarios.
\end{abstract}

Palabras clave: Salud de la persona mayor; Marcadores bioquímicos; Profesor universitario. (fuente: DeCS BIREME)

\begin{abstract}
Objective: Compare the values of the blood biomakers: uric acid, creatinine, glucose, cholesterol and triglycerides, in two groups of university professors apparently healthy. Methods: The first group, formed by 58 professors aged 60 to 64 , and the second formed by 35 professors aged 75 to 81 , in order to evaluate if there is a significant statistic difference in both groups. Results: The values of uric acid in group I were between 2,50 mg/dL. and 8,20 $\mathrm{mg} / \mathrm{dL}$; in groups II were between $2,50 \mathrm{mg} / \mathrm{dL}$ and $8,63 \mathrm{mg} / \mathrm{dL}$, showing a significant difference $(\mathrm{p}=0.035)$. The values of creatinine in group I were between $0,64 \mathrm{mg} / \mathrm{dL}$ and 1,56 mg/dL; and in group II were between $0,80 \mathrm{mg} /$ $\mathrm{dL}$ and $1,61 \mathrm{mg} / \mathrm{dL}$., showing a significant difference $(\mathrm{p}=0.004)$. The values of glucose in group I were between 63 $\mathrm{mg} / \mathrm{dL}$ and $188 \mathrm{mg} / \mathrm{dL}$; and in group II were between $60 \mathrm{mg} / \mathrm{dL}$ y $297 \mathrm{mg} / \mathrm{dL}$, not showing significant difference $(p=0.671)$. The values of cholesterol in group I were between $125 \mathrm{mg} / \mathrm{dL}$ and $265 \mathrm{mg} / \mathrm{dL}$; and in group II were between $107 \mathrm{mg} / \mathrm{dL}$ and $244 \mathrm{mg} / \mathrm{dL}$; not showing significant difference $(p=0.053)$. The values of triglycerides in group I were between $58 \mathrm{mg} / \mathrm{dL}$ and $507 \mathrm{mg} / \mathrm{dL}$; and in group II were between $68 \mathrm{mg} / \mathrm{dL}$ and $314 \mathrm{mg} / \mathrm{dL}$; not showing significant difference $(p=0.104)$. Conclusion: There is a significant difference in the biomarkers of uric acid and creatinine between the age groups; however, there was not found significant difference in the biomarkers of glucose, cholesterol and triglycerides between the age groups.
\end{abstract}

Key words: Health of the elderly; Biochemical marker; University professor. (source: MeSH NLM)

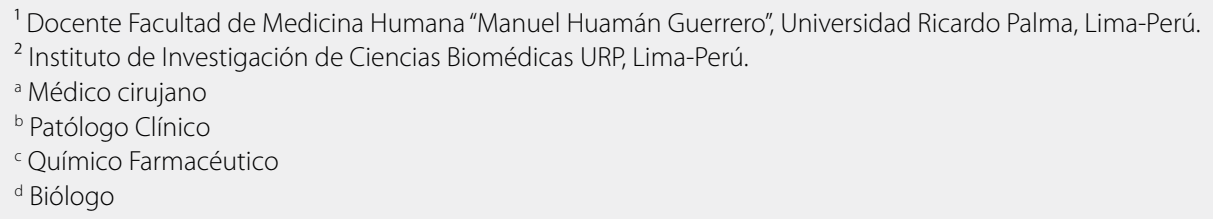

Citar como: Nicanor Domínguez-Navarrete, Cecilia Rojas-Guerrero, Carola Chambers-Medina. Estudio comparativo de marcadores bioquímicos de salud, en dos grupos etarios de docentes universitarios, 2017. [Artículo Original].2019;19(2):00-00. (Abril 2019). DOl 10.25176/RFMH.v19.n2.2063 


\section{INTRODUCCIÓN}

Para evaluar el estado de salud de una persona, los médicos suelen incorporar al examen clínico general procedimientos que se denominan "ayuda al diagnóstico". Un grupo de ellos son los exámenes de laboratorio, que permiten determinar el nivel de ciertas sustancias bioquímicas en la sangre periférica del paciente, y que son expresión del estado funcional de un órgano determinado. Para ello, se han elaborado tablas, con cifras de los niveles de dichas sustancias bioquímicas en el rango mínimo y máximo, con las unidades de medida correspondiente; así mismo, en estas tablas figuran las variables de normalidad'.

Para interpretar el significado clínico del valor de una sustancia bioquímica que está fuera del rango establecido como normal, debe considerarse, obligatoriamente, el contexto de la historia clínica del paciente en particular.

Los marcadores bioquímicos seleccionados en este estudio son: ácido úrico, creatinina, glucosa, colesterol y triglicéridos. Se hará una explicación sucinta de la importancia de cada una de ellos:

El ácido úrico, es un compuesto químico producto final del catabolismo de las purinas, no es metabolizado por las células humanas, y en la sangre se ioniza como urato monosódico. Los excedentes forman cristales ocasionando el síndrome clínico denominado Gota.

La creatinina es un producto del catabolismo de proteínas del músculo estriado, que se excreta por filtración glomerular, por lo cual, es muy útil para valorar la función renal.

La glucosa, es el compuesto bioquímico fuente de energía del metabolismo celular. El nivel sanguíneo de glucosa es regulado por la insulina, la función de la membrana citoplasmática celular y el metabolismo del tejido adiposo. La alteración de estos mecanismos ocasiona la Diabetes Mellitus, expresado por hiperosmolaridad endovascular y daño del endotelio vascular.

El colesterol es un compuesto lípido esteroideo, base para el origen de los ácidos biliares, hormonas esteroideas y vitamina D. Es una molécula esencial para la membrana citoplasmática de las células humanas. Los excesos de este compuesto se relacionan con la obesidad y la ateroesclerosis.

Los triglicéridos, son moléculas formadas por la glicina con los tres hidroxilos esterificados con ácidos grasos. Son compuestos de almacenamiento de los lípidos y son metabolizados en el hígado y células adiposas.
Los niveles elevados ocasionan dislipidemias, con compromiso del endotelio vascular.

En las últimas décadas, la tendencia a realizarse exámenes médicos con criterio preventivo es cada vez más frecuente. Incluso, es obligatorio cuando se ingresa a ciertas entidades laborales. La Ley de trabajo ${ }^{2}$ establece la evaluación bianual, realizada por un médico especialista en Medicina Ocupacional.

La población docente de la Universidad Ricardo Palma está constituida por un porcentaje importante de adultos mayores (más de 65 años), y se piensa que un porcentaje de ellos puedan tener cifras alteradas de los marcadores bioquímicos usados para evaluar salud, por lo que existe una preocupación velada en este grupo experimentado de docentes, de tener algún marcador bioquímico con cifras fuera de lo normal, y ser ésta información una razón de alejamiento de sus actividades docentes. Por ello, se ha elaborado el presente estudio, con el objetivo de comparar la mediana de éstos valores, la tendencia hacia cifras superiores del valor normal, y si hay diferencia estadística de los marcadores bioquímicos entre las dos poblaciones de docentes aparentemente normales: una adulta madura de 60 a 64 años de edad y otra con vejez intermedia de 75 a 81 años.

\section{MÉTODOS}

El presente estudio es de tipo transversal, observacional y descriptivo. Los participantes corresponden a los docentes que concurrieron a la convocatoria para la evaluación de Salud Ocupacional, durante el segundo semestre del año 2016. Las muestras se procesaron en el Laboratorio Clínico del Policlínico FAMURP de la Facultad de Medicina Humana "Manuel Huamán Guerrero", Universidad Ricardo Palma. Los requisitos de inclusión fueron: a) No tener sintomatología alguna, ni diagnóstico previo de enfermedad, y b) tener la edad comprendida entre 60 a 64 años (adulto maduro) y de 75 a 81 años (vejez intermedia).

Para los exámenes bioquímicos sanguíneos los pacientes fueron citados en ayunas. El proceso se realizó en el analizador "Chamry", con reactivos de metodología enzimática. Para determinar ácido úrico se empleó la marca "Elitech", con rangos de normalidad de 3,5 a $7,2 \mathrm{mg} / \mathrm{dL}$ en hombres y 2,6 a $6,0 \mathrm{mg} / \mathrm{dL}$ en mujeres. Para determinar creatinina se empleó el picrato alcalino de la marca "Valtek", con rangos de normalidad de 0,7 a 1,4 mg/dL. En hombres y 0,6 a 1,2 $\mathrm{mg} / \mathrm{dL}$. En mujeres. Para determinar glucosa se empleó la marca "Elitech", con rangos de normalidad de 75 a $110 \mathrm{mg} / \mathrm{dL}$. Para determinar colesterol se usó la marca 
"Elitech", con rangos de normalidad de 150 a 200 mg/ dl. Para determinar triglicéridos se empleó la marca "Elitech", con rangos de normalidad inferior a $150 \mathrm{mg} /$ $\mathrm{dL}$ en hombres e inferior a $130 \mathrm{mg} / \mathrm{dL}$ en mujeres.

El análisis estadístico, en el presente estudio se utilizó el programa Excel para el almacenamiento de datos; las fórmulas necesarias para obtener la distribución y dispersión de los datos; y se utilizó la Prueba $U$ de Mann Whitney para obtener la diferencia significativa, a nivel 0,05, entre ambos grupos.

\section{RESULTADOS}

Durante la evaluación asistieron 58 docentes con las edades comprendidas entre los 60 a 64 años (15 correspondieron al sexo femenino y 43 al masculino), que formaron el grupo I. Asimismo, asistieron 35 docentes con las edades comprendidas entre 75 a 81 años (3 correspondieron al sexo femenino y 32 al masculino), que formaron el grupo II. Tabla 1.

El nivel de ácido úrico en los docentes del grupo l estuvo entre $2,50 \mathrm{mg} / \mathrm{dL}$. y $8,20 \mathrm{mg} / \mathrm{dL}$, con una mediana de $4,68 \mathrm{mg} / \mathrm{dL}$. En el grupo II, el nivel estuvo entre $2,50 \mathrm{mg} /$ $\mathrm{dL}$ y $8,63 \mathrm{mg} / \mathrm{dL}$, con una mediana de $4,00 \mathrm{mg} / \mathrm{dL}$. Se detectó un caso, en cada grupo con cifra superior a lo normal, representando el $1.7 \%$ y $2.8 \%$ respectivamente. Tablas 2 y 3 . Figura 1.

El nivel de creatinina en el grupo l, estuvo entre 0,64 $\mathrm{mg} / \mathrm{dL}$ y $1,56 \mathrm{mg} / \mathrm{dL}$, con una mediana de $1,05 \mathrm{mg} / \mathrm{dL}$. En el grupo II, el nivel estuvo entre 0,80 mg/dL y 1,61 $\mathrm{mg} / \mathrm{dL}$., con una mediana de 1,20 mg/dL. Se detectó a docentes con cifras superiores a lo normal, así: cuatro casos en el grupo I, que representan el 6,8\%, y cinco casos en el grupo II, que representan el 14,2\%. Tablas 2 y 3. Figura 2.

El nivel de glucosa en el grupo I estuvo entre $63 \mathrm{mg} /$ $\mathrm{dL}$ y $188 \mathrm{mg} / \mathrm{dL}$, con una mediana de $92.50 \mathrm{mg} / \mathrm{dL}$. En el grupo II, el nivel estuvo entre $60 \mathrm{mg} / \mathrm{dL}$ y $297 \mathrm{mg} /$ $\mathrm{dL}$, con una mediana de $94 \mathrm{mg} / \mathrm{dL}$. En ambos grupos se encontró a docentes con cifras superiores a lo normal, así: siete casos en el grupo l, que representan el 12.0\%, y seis casos en el grupo II, que representan el $17.1 \%$. Tablas 2 y 3 . Figura 3.

El nivel de colesterol en el grupo I estuvo entre $125 \mathrm{mg} /$ $\mathrm{dL}$ y $265 \mathrm{mg} / \mathrm{dL}$, con una mediana de $196.50 \mathrm{mg} / \mathrm{dL}$. En el grupo II, el nivel estuvo entre 107 mg/dL y $244 \mathrm{mg} /$ $\mathrm{dL}$., con una mediana de $182 \mathrm{mg} / \mathrm{dL}$. En ambos grupos se encontró a docentes con cifras superiores a lo normal, así: 24 casos en el grupo l, que representan el 41,3\%, y 10 casos en el grupo II, que representan el $28,5 \%$. Tablas 2 y 3 . Figura 4.

El nivel de triglicéridos en el grupo I estuvo entre 58 mg/dL y 507 mg/dL., con una mediana de 147.50 mg/ $\mathrm{dL}$. En el grupo II, el nivel estuvo entre $68 \mathrm{mg} / \mathrm{dL}$ y 314 $\mathrm{mg} / \mathrm{dL}$, con una mediana de $132 \mathrm{mg} / \mathrm{dL}$, En ambos grupos se encontró a docentes con cifras superiores a lo normal, así: 31 casos en el grupo l, que representan el $53,4 \%$, y 10 casos en el grupo II, que representan el $28,5 \%$. Tablas 2 y 3 . Figura 5 .

\section{DISCUSIÓN}

La cifra de ácido úrico en sangre depende, en gran medida, de la dieta hiperproteica y/o a factores endógenos, que permiten su incremento. Aunque la mayoría de los docentes estudiados tienen cifras en el rango de la normalidad, sólo encontramos a un docente en cada grupo con cifra superior al normal $(8,20$ y $8,63 \mathrm{mg} / \mathrm{dL}$ respectivamente). El grupo I muestra un ligero incremento, que la Prueba estadística $\mathrm{U}$ de Mann Whitney, tabla 4, la considera como diferencia significativa entre ambos grupos $(p=0.035)$. Posiblemente la población con vejez intermedia tenga un régimen alimenticio conservador, que explique los niveles menores de ácido úrico. Hay que señalar, que el estudio de Cheng W. ${ }^{3}$ demuestra una relación significativa entre hiperuricemia e hipertensión arterial, en una población de 1.082 personas entre 41 a 70 años. Asimismo, Luo DL. ${ }^{4}$, señala a la hiperuricemia como factor predictivo de casos severos de hipertensión pulmonar, entidades frecuentes en adultos mayores.

Tabla 1. Distribución de la muestra de docentes universitarios según grupos de comparación y sexo.

\begin{tabular}{lccc}
\multicolumn{1}{r}{ SEXO } & MUESTRA & GRUPOS \\
& & GRUPO I & GRUPO II \\
\hline Masculino & 75 & 43 & 32 \\
Femenino & 18 & 15 & 3 \\
\hline
\end{tabular}


Tabla 2. Estadísticos descriptivos de los marcadores bioquímicos, según grupos de comparación.

\begin{tabular}{|c|c|c|c|c|c|}
\hline GRUPOS DE COMPARACIÓN & MíN. & MÁX. & MEDIA & DESV. EST. & MEDIANA \\
\hline \multicolumn{6}{|l|}{ Grupo I } \\
\hline Ácido úrico (mg/dl) & 2,50 & 8,20 & 4,89 & 1,34 & 4,68 \\
\hline Creatinina (mg/dl) & 0,64 & 1,56 & 1,05 & 0,22 & 1,05 \\
\hline Glucosa (mg/dl) & 63,00 & 188,00 & 96,45 & 20,33 & 92,50 \\
\hline Colesterol (mg/dl) & 125,00 & 265,00 & 196,47 & 36,22 & 196,50 \\
\hline Triglicéridos (mg/dl) & 58,00 & 507,00 & 167,88 & 89,64 & 147,50 \\
\hline \multicolumn{6}{|l|}{ Grupo II } \\
\hline Ácido úrico (mg/dl) & 2,50 & 8,63 & 4,36 & 1,50 & 4,00 \\
\hline Creatinina (mg/dl) & 0,80 & 1,61 & 1,21 & 0,25 & 1,20 \\
\hline Glucosa (mg/dl) & 60,00 & 297,00 & 106,77 & 49,23 & 94,00 \\
\hline Colesterol (mg/dl) & 107,00 & 244,00 & 180,09 & 35,03 & 182,00 \\
\hline Triglicéridos (mg/dl) & 68,00 & 314,00 & 138,71 & 58,39 & 132,00 \\
\hline
\end{tabular}

Tabla 3. Cuadro comparativo de medianas de marcadores bioquímicos y los valores anormales entre ambos grupos.

\begin{tabular}{|c|c|c|c|c|c|c|}
\hline \multirow{3}{*}{ MARCADORES BIOQUÍMICOS } & \multicolumn{3}{|c|}{ GRUPO I } & \multicolumn{3}{|c|}{ GRUPO II } \\
\hline & \multirow[t]{2}{*}{ MEDIANA } & \multicolumn{2}{|c|}{ ANORMAL } & \multirow[t]{2}{*}{ MEDIANA } & \multicolumn{2}{|c|}{ ANORMAL } \\
\hline & & $\mathbf{N}^{\circ}$ & $\%$ & & $\mathbf{N}^{\circ}$ & $\%$ \\
\hline Acido úrico (mg/dL) & 4,68 & 1 & 1,7 & 4.00 & 1 & 2,86 \\
\hline Creatinina (mg/dL) & 1,05 & 4 & 6,8 & 1,20 & 5 & 14,2 \\
\hline Glucosa (mg/dL) & 92,5 & 7 & 12 & 94 & 6 & 17 \\
\hline Colesterol (mg/dL) & 196 & 24 & 41,3 & 182 & 10 & 28,5 \\
\hline Triglicérido (mg/dL) & 147,5 & 31 & 53,4 & 132 & 10 & 28,5 \\
\hline
\end{tabular}

Tabla 4. Prueba U de Mann Whitney para los marcadores bioquímicos según grupos de comparación.

\begin{tabular}{|c|c|c|c|c|}
\hline $\begin{array}{l}\text { MARCADORES } \\
\text { BIOQUÍMICOS }\end{array}$ & $\begin{array}{l}\text { GRUPOS DE } \\
\text { COMPARACIÓN }\end{array}$ & RANGO PROMEDIO & $\begin{array}{l}\text { U DE MANN } \\
\text { WHITNEY }\end{array}$ & P VALOR \\
\hline \multicolumn{5}{|l|}{ Ácido úrico (mg/dl) } \\
\hline & Grupo I & 51,59 & \multirow{2}{*}{748,500} & \multirow{2}{*}{$0,035 *$} \\
\hline & Grupo II & 39,39 & & \\
\hline \multicolumn{5}{|l|}{ Creatinina (mg/dl) } \\
\hline & Grupo I & 40,83 & \multirow{2}{*}{657,000} & \multirow{2}{*}{$0,004 *$} \\
\hline & Grupo II & 57,23 & & \\
\hline \multicolumn{5}{|l|}{ Glucosa (mg/dl) } \\
\hline & Grupo I & 46,08 & \multirow{2}{*}{961,500} & \multirow{2}{*}{0,671} \\
\hline & Grupo II & 48,53 & & \\
\hline \multicolumn{5}{|l|}{ Colesterol (mg/dl) } \\
\hline & Grupo I & 51,20 & \multirow{2}{*}{771,500} & \multirow{2}{*}{0,053} \\
\hline & Grupo II & 40,04 & & \\
\hline \multicolumn{5}{|l|}{ Triglicéridos (mg/dl) } \\
\hline & Grupo I & 50,53 & \multirow{2}{*}{810,000} & \multirow{2}{*}{0,104} \\
\hline & Grupo II & 41,14 & & \\
\hline
\end{tabular}

*Significativo o a un nivel de 0,05 


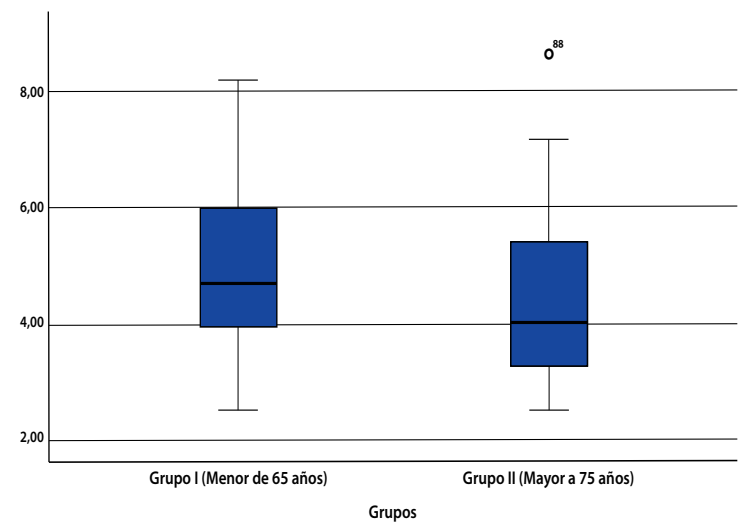

Gráfico 1. Niveles de ácido úrico $(\mathrm{mg} / \mathrm{dl})$ según grupos de comparación.

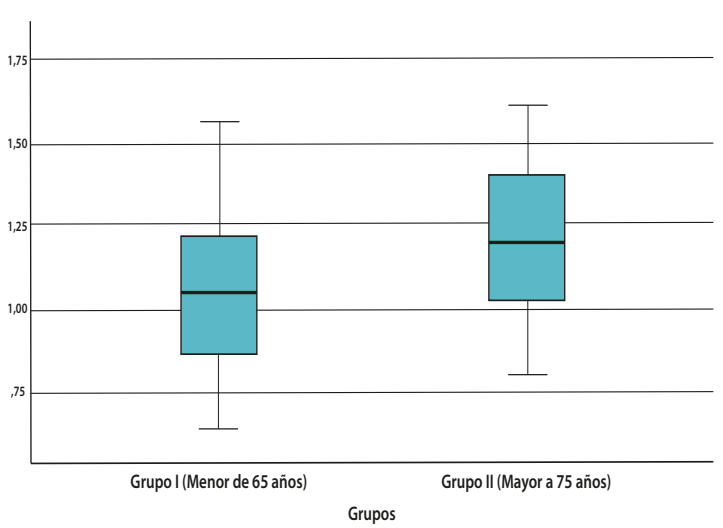

Gráfico 2. Niveles de creatinina (mg/dl) según grupos de comparación.

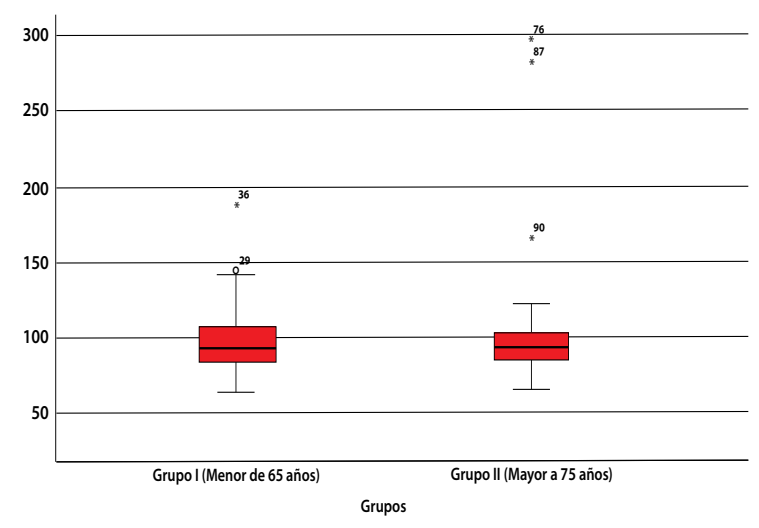

Gráfico 3. Niveles de glucosa (mg/dl) según grupos de comparación.

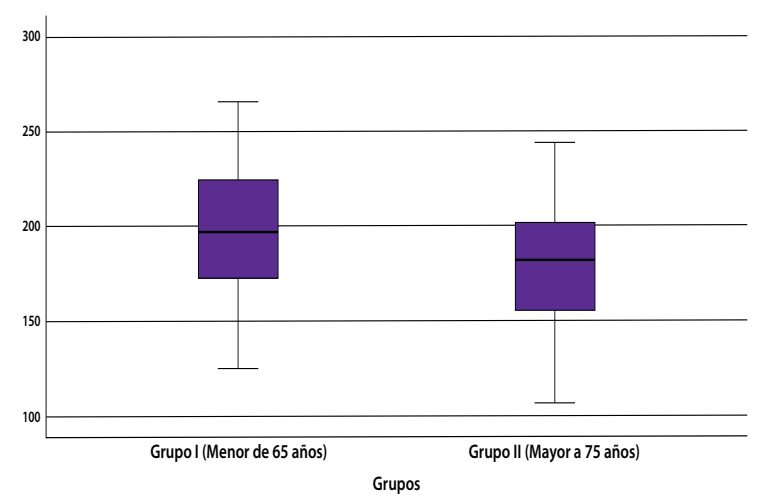

Gráfico 4. Niveles de glucosa $(\mathrm{mg} / \mathrm{dl})$ según grupos de comparación.

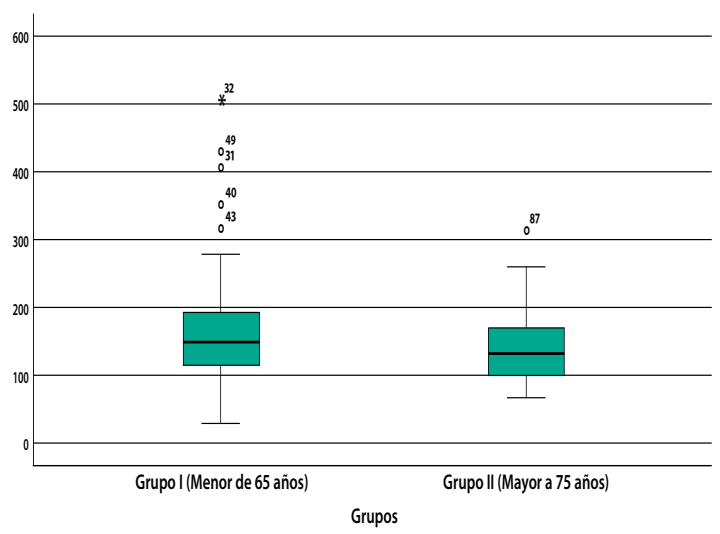

Gráfico 5. Niveles de triglicéridos (mg/dl) según grupos de comparación.

La creatinina, es un metabolito cuyo nivel sanguíneo es bastante estable en el curso del tiempo, por lo tanto, es un buen marcador utilizado en clínica para evaluar función renal. La mediana de creatinina, en ambos grupos, se encuentra en el rango normal; aunque los valores del grupo Il son $20 \%$ superiores, con un máximo de $1,6 \mathrm{mg} / \mathrm{dL}$. Este pequeño incremento en el grupo II tiene valor estadístico $(p=0.004)$ en la Prueba U de Mann Whitney, tabla 4. Nuestros resultados se encuentran en acorde con lo señalado por Sebastiani P. ${ }^{5}$, que luego de evaluar a 5.000 adultos mayores longevos, encuentra que el nitrógeno no proteico y el systatin (proteína que mide filtración glomerular) se incrementan con la edad.

Las medianas de la cifra de glucosa en sangre de ambos grupos es similar, y no hay diferencia estadística $(p=0.671)$, tabla 4 . Aunque se encontraron en el grupo 
I a siete docentes (12\%) y en el grupo II a seis docentes (17\%) con niveles de glucosa superiores a lo normal; nivel que obliga a realizar estudios complementarios para el diagnóstico de Diabetes Mellitus (DM). La comunicación del Villena $\mathrm{JE}^{6}$ menciona, que la DM afecta al $7 \%$ de la población en el Perú, y que la prevalencia del síndrome metabólico en los ancianos es mucho mayor que en otros grupos humanos.

El nivel sanguíneo de colesterol es una preocupación frecuente de los adultos mayores, pues está en relación directa con la obesidad 7 , la hipertensión arterial8, la osteoporosis ${ }^{9}$ y el estado del endotelio vascular. Las medianas de la cifra de colesterol en el grupo I y || están en el rango de la normalidad, y no hay diferencia estadística ( $p=0.053$ ) en la Prueba U de Mann Whitney, tabla 4. Pero, 24 (41,3\%) docentes del grupo I, y $10(28,5 \%)$ del grupo II, tienen cifras de colesterol superiores al rango normal; posiblemente en relación a hábitos alimenticios comunitarios. Aunque, Zanchetti A. ${ }^{8}$, evaluando a 5.376 pacientes mayores de 64 años, encuentra prevalencia de hipercolesterolemia con hipertensión arterial, razón que justifica el conocer la cifra sanguínea de colesterol.

Los triglicéridos que se determinan en sangre periférica son, en su mayoría, procedentes de la ingesta diaria ${ }^{10}$, por ello, las cifras elevadas de triglicéridos son expresión de desorden alimenticio, que conlleva a la dislipidemia. Las medianas de triglicéridos, en ambos grupos, están debajo del límite superior normal, y no hay diferencia estadística significativa $(p=0.104)$ en la Prueba $U$ de Mann Whitney, tabla 4. Aun así, se encontraron, en el grupo I, a 31 (53.4\%) docentes, y en el grupo II, a $10(28,5 \%)$ de docentes con cifras superiores a lo normal. La importancia clínica del conocimiento del nivel sanguíneo de triglicéridos en adultos mayores es válida, además del síndrome de dislipidemia, es la malnutrición, como lo señala Amador-Licona N. ${ }^{11}$ que encuentra el $93 \%$ de malnutrición en adultos mayores (65 a 85 años) con fractura de cadera, y poca fuerza muscular, asociados al síndrome metabólico.
Por otro lado, Choi HJJ12, evaluó la actividad cognitiva de 59 adultos mayores que tenían hipertrigliceridemia, encontrando cierta asociación, que sugiere que el nivel elevado de triglicéridos juega algún rol en la amiloidosis cerebral.

La evaluación de salud que se realiza al trabajador docente mide el estado funcional de diversos órganos, pero no mide el grado de compromiso de las cualidades mentales, necesarias para ejercer una buena docencia.

\section{CONCLUSIÓN}

En los marcadores bioquímicos de ácido úrico y creatinina, hay diferencia significativa entre ambos grupos etarios. Las cifras de ácido úrico son superiores en el grupo I, en cambio, las cifras de creatinina son superiores en el grupo II. A pesar de que la mayoría de las determinaciones estuvieron, en ambos grupos, en el rango normal.

En los marcadores de glucosa, colesterol y triglicéridos, no se demuestra diferencia significativa entre ambos grupos etarios. A pesar del porcentaje de cifras superiores a lo normal que se observa: el 12\%, 41.3\% y $53.4 \%$ respectivamente, en el grupo l; y el $17 \%, 28.5 \%$ y $28.5 \%$ respectivamente, en el grupo II.

Contribuciones de autoría: Los autores participaron en la generación, redacción y aprobación final del artículo original.

Financiamiento: Autofinanciado.

Conflicto de interés: Los autores declaran no tener conflicto de intereses en la publicación de este artículo.

Recibido: 07 de octubre de 2018

Aprobado: 19 de diciembre de 2018

Correspondencia: Nicanor Domínguez Navarrete Dirección: Calle Enrique Olivero 268 San Borja, Lima-Perú Teléfono: +51998886405

Correo:ndominguez38@yahoo.com 


\section{REFERENCIAS BIBLIOGRÁFICAS}

1. K.D. Pagana, T.J. Pagana. Guía de pruebas diagnósticas y de laboratorio. Alsevier 8va. Edición, 2008

2. Ley del trabajo $N^{\circ} 29783$, y su modificatoria №30222, Artículo 49.

3. Cheng W, Wen S, Wang Y, Tan Y, Li H, Hou Y, Hu H, Golledge J, Yang $\mathrm{G}$. The association between serum uric acid and blood pressure in different age groups in healthy Chinese cohort. Medicine (Baltimore). 2017 Dec 96(50): e8953. Disponible en: www.ncbi.nlm.nih.gov/ pubmed/29390287

4. Luo DL, Zhang CJ, Huang YG, Huang T, Li HZ. Serum uric acid is associated with disease severity and an important predictor for clinical outcome in patients with pulmonary hypertension. Zhonghua Xin Xue Guan Bing Za Zhi. 2017 Jan24;45(6):496-500.

5. Sebastiani P, Thyagarajan B, Sun F, Honig LS, Schupf N, Cosentino S, Feitosa MF, Wojczynski M, Newman AB, Montano M, PerlTT. Age and sex distributions of age-related biomarker values in healthy older adults from the long life family study. J AM Geriatr Soc. 2016 Nov 64:e189-e194. $\Rightarrow$ Disponible en: www.nsbi.nlm.nih.gov/pubmed/27783390

6. Villena JE. Diabetes Mellitus in Perú. Ann Glob. Health. 2015 Nov Dec;81(6):765-75.

7. Llanos Zavalaga Fernando; Nájar Trujillo Néstor; Mayca Pérez Julio Rosas Aguirre Angel. Prevalencia de obesidad e hipercolesterolemia en la Facultad de Medicina de la Universidad Peruana Cayetano Heredia 1988. Rev Med Hered Vol. 12 N.3 Lima ene-jul. 2001.

8. Zanetti A. Hyperlipidemia in the hypertensive patient. Am J Med. 1994 Jun6; 96(6A):3S-8S. Disponible en: www.ncbi.nih.gov/ pubmed/27108144

9. Wang Y, Dai J, Zhong W, Hu C, Lu S, Chai Y. Association between serum cholesterol level and osteoporotic fractures. Front endocrinol (Lausanne). 2018 Feb 12;9:30. Disponible en: www.ncbi.nlm.nih.gov/ pubmed/29483896

10. Feflova VV, Koloskova TP, Kazakova TV, Fefelova YA-. Alteration of serum lipid profile in young men with different somatotypes after food load. Vopr Pitan. 2015;84(1):25-30

11. Amador-Licona N, Moreno-Vargas EV, Martínez-Cordero C. Protein intake, serum lipids and muscle strenght in the elderly. Nutr Hosp 2018 Jan 10;35(1):65-70. Disponible en: www.ncbi.nlm.nih.gov/ pubmed/29565151

12. Choi HJ, Byun MS, Yi D, Choe YM, Sohn BK, Baek HW, Lee JH, Kim HJ, Han JY, Yoon EJ, Kim YK, Woo Jl, Lee DY. Association between serum triglycerides and cerebral amyloidosis in cognitively normal elderly. Amm J Geriatr Psychiatry. 2016 Aug;24(8):604-612. Disponible en: www. ncbi.nlm.nih.gov/pubmed/27311886

\section{latindex}

http://www.latindex.org/latindex/ficha?folio=14280

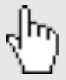

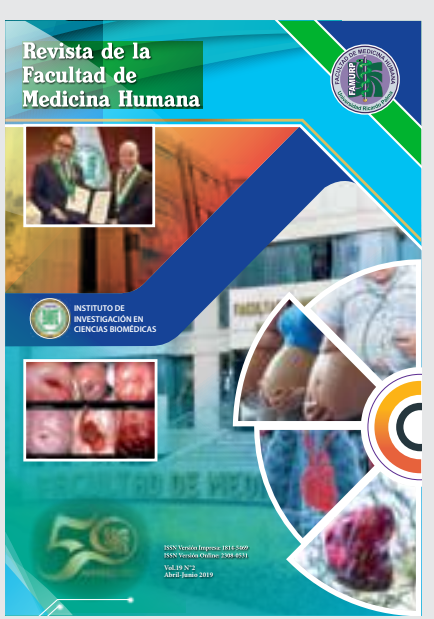

